Abstract AB1084 - Table 1

\begin{tabular}{lccc}
\hline & $\begin{array}{c}\text { Calprotectin }(\mu \mathrm{g} / \mathrm{mL}) \\
\text { (range) }\end{array}$ & $\begin{array}{c}\text { PCR }(\mathrm{mg} / \mathrm{dL}) \\
\text { (range) }\end{array}$ & $\begin{array}{c}\text { ESR }(\mathrm{mmHg}) \\
\text { (range) }\end{array}$ \\
\hline Active & $3,43^{1,80-5,31}$ & $4,27(0,7-11,6)$ & $16,86^{3-51}$ \\
Inactive & $2,15(0,78-4,06)$ & $1,83(0,7-4,6)$ & $13,11^{3-43}$ \\
\hline
\end{tabular}

Calprotectin was statistically correlated with Clinical Activity $(p=0.018)$, however, neither ESR ( $p=0.539)$ nor RCP $(p=0.059)$ did, although in RCP there was a clinical trend, (ANOVA)

Calprotectin, RCP and ESR were negative in $91 \%, 80 \%$ and $76 \%$ respectively of Inactive patients; and positive in $43 \%, 100 \%$ and $33 \%$ of the Active ones

The analysis of the ROC curves in our sample showed that the value that allows to discriminate between active and non-active disease with a Sensitivity of $80 \%$ and a Specificity of $69 \%$ is $2 ' 07 \mu \mathrm{g} / \mathrm{mL}$

Serum Calprotectin was 2 points higher in the group of patients with Autoinflammatory diseases than in the group of JIA, with a mean of 4.91 compared to 2.90 $(p=0.002)$. However, since it is a retrospective study, we must bear in mind that this can be influenced by the reasons for the test request, being in the group of Autoinflammatory Disease the suspicion of active disease, and in the AIJ simply monitoring or assessment of treatment optimisation.

It should be noted that, in the patients in diagnostic process that did not present any rheumatological disease (final diagnoses of: arthralgias in 3 cases and glomerulonephritis not associated to rheumatologic/autoimmune disease in 1), serum Calprotectine did not exceed in any case the $1.15 \mu \mathrm{g} / \mathrm{mL}$

Conclusions: Serum Calprotectin is emerging as a useful marker, not only in the field of JIA, but also in other diagnostic groups such as Autoinflammatory Diseases.

Prospective and larger studies are needed to determine its role

Disclosure of Interest: None declared

DOI: 10.1136/annrheumdis-2018-eular.3529

\section{AB1085 THE FACTORS AFFECTING REMISSION IN JIA PATIENTS, SINGLE CENTRE RESULTS}

B. Sozeri ${ }^{1}$, T. Demircan Bilen ${ }^{1}$, D. Kurtulus ${ }^{2}$, I. Islek ${ }^{1} .{ }^{1}$ Pediatric Rheumatology; ${ }^{2}$ Physical Medicine and Rehabilitation, University of Health Sciences, Istanbul, Umraniye Training and Research Hospital, Istanbul, Turkey

Background: Juvenile idiopathic arthritis (JIA) is the most common rheumatic disease in childhood. Nowadays, in the management of JIA, clinical remission is the accepted goal. However, remission is much associated with the JIA subtype and inversely with suggested predictors for poor outcome.

Objectives: The aim of the study is to evaluate the predictors of clinical outcome in patients with juvenile idiopathic arthritis

Methods: This was a single-centre, an observational study including children diagnosed with JIA at Health Sciences University Istanbul, Umraniye Education and Research Hospital, Turkey, between June 2016 and January 2018. All patients were evaluated at the time of diagnosis, and at 3 months of their disease. We routinely collected the patients' baseline profile which included the age of disease onset, gender, number of active joints, patients'and physicians'global assessment of disease activity (PGA, range from 0 to $10 \mathrm{~mm}$; 0 is the best score), the JADAS 27 score. and therapy. Patients were identified through the divisional database which includes all patients seen in the rheumatology clinic.

Results: A total of $141 \mathrm{JIA}$ patients were included in the analysis. The mean follow-up duration of these patients was 23.36 months (range from 12 to 156 months). We examined 71 joints in all patients. At the time of diagnosis, it was seen that they were frequently in knee joint $(61.7 \%)$ and sacroiliac joints (34.8\%). The rate of improvement of joints in the last visit was found to be metatarsophalangeal joints (100\%), sacroiliac joints (79\%) and knee joints (44.8\%), respectively. The non-systemic group $(n=133)$ was evaluated for initial steroid use, among the patients, $54(\% 41)$ had steroid therapy at the time of diagnosis. There was seen that 29 of the 141 patients $(20.6 \%)$ were in the remission in 3 months of follow-up. According to subtype of JIA patients, systemic patients (62.5\%) were frequently in remission at 3 months. At the end of the study, of the 141 patients, $72(51.1 \%)$ achieved remission and were assigned to the remission group. Among the patients in the remission group, $44(49.4 \%)$ had episodes of disease flares. The other $30(40.5 \%)$ patients did not have disease flares. Comparing the baseline data in the remission group and non-remission group, there was no difference between these two groups in disease onset age, gender, JIA subtypes, number of active joints at disease onset and JADAS 27 scores and used steroid therapy. We also analysed whether each group used biological drugs and analysed the duration of starting biologic therapy. Among 72 patients achieved remission, 17 $(23.6 \%)$ patients had at least one biological drug. There was no difference between two groups. The median time of biologic drug starting was 12 (interquartile range (IQR), 12) months. There was longer time of biologic drug starting in non-remission group than remission group (32.3 \pm 36.2 vs $25.0 \pm 31.4, p>0,05)$. We determine that in systemic and poli JIA patients who were used biological therapy were found to have a high percentage of achieved to remission

Conclusions: JIA is a heterogeneous disease with significant variability in course and long-term outcome. No parameter could be used as a single predictor of long term outcomes. Standardised baseline work-up, disease activity evaluation and a definition of a treat to target approach will result in better health outcomes for JIA patients

Disclosure of Interest: None declared

DOI: 10.1136/annrheumdis-2018-eular.4234

\section{AB1086 A 6-MONTH, MULTICENTER, OPEN-LABEL, EXPLORATORY STUDY OF FIXED DOSE NAPROXEN/ ESOMEPRAZOLE IN ADOLESCENT PATIENTS WITH JUVENILE IDIOPATHIC ARTHRITIS (JIA)}

D.J. Lovell ${ }^{1}$, J.A. Dare ${ }^{2}$, J. Ball ${ }^{3}$, M. Francis-Sedlak ${ }^{3}$, B.D. LaMoreaux ${ }^{3}$, R.J. Holt ${ }^{3,4}$ ${ }^{1}$ Cincinnati Children's Hospital Medical Center, Cincinnati; ${ }^{2}$ Arkansas Children's Hospital, Little Rock; ${ }^{3}$ Horizon Pharma USA, Inc., Lake Forest, ${ }^{4}$ University of Illinois-Chicago, College of Pharmacy, Chicago, USA

Background: JIA is an inflammatory arthritis of unknown etiology, which lasts for greater than 6 weeks with onset before 16 years of age. ${ }^{12}$ Per the ACR guidelines, JIA is the most common chronic rheumatic disease in children. ${ }^{3}$ NSAIDs have been the mainstay of initial management with naproxen being commonly used, but they can cause serious side effects such as gastritis. Herein, we report the results of a clinical trial in JIA patients receiving one of 3 fixed doses of naproxen/esomeprazole magnesium (nap/eso) given BID (table 1).

Objectives: To evaluate the signs/symptoms of JIA, safety and tolerability of nap/ eso in adolescents age 12 to 16 years.

Methods: Forty-six children and adolescents with JIA by ILAR criteria, mean age of 13.6 years, from 18 US sites were prospectively enrolled over 2 years and treated for up to 6 months. Mean number of active joints at baseline was 3.1 Doses were based on baseline weight (table 1). Disease activity was assessed with the ACR Pediatric-30,-50,-70,-90 Response and the Childhood Health Assessment Questionnaire (CHAQ) discomfort and functional scores at months 1,3 , and 6 as change from baseline. Occurrence and causality were assessed for treatment emergent AEs (TEAEs) and discontinuations were monitored monthly. Results: Forty-six patients received at least 1 dose of study drug and $36 \mathrm{com}$ pleted the trial. The\% of patients achieving ACR Paediatric response increased over time (figure 1). CHAQ discomfort improved at each assessment and functional scores improved at all assessments for 'Arising, Walking, and Activities' with several improved for 'Dressing and Grooming, Eating, Hygiene, and Grip'. There was no indication of a dose-related efficacy effect. Thirty-seven (80.4\%) had at least 1 TEAE. Frequent TEAEs $(\geq 5 \%)$ were upper respiratory tract infection, upper abdominal pain, sinusitis, diarrhoea, headache, nausea, and ligament sprain. Eleven $(23.9 \%)$ had at least 1 TEAE considered to be related to study drug. Most frequent study drug-related TEAE ( $\geq 5 \%$ ) was upper abdominal pain Four (8.7\%) discontinued due to a TEAE.

Abstract AB1086 - Table 1. Minimum and Maximum Study Drug Dose (nap/eso) by Weight Group

\begin{tabular}{lrr}
\hline Weight at Enrollment $\mathbf{( k g})^{1}$ & Minimum Dose $^{2}$ & Maximum dose $^{2}$ \\
\hline$<38$ & $250 \mathrm{mg} / 20 \mathrm{mg}$ & $250 \mathrm{mg} / 20 \mathrm{mg}$ \\
$38-<50$ & $250 \mathrm{mg} / 20 \mathrm{mg}$ & $375 \mathrm{mg} / 20 \mathrm{mg}$ \\
$50-<75$ & $375 \mathrm{mg} / 20 \mathrm{mg}$ & $500 \mathrm{mg} / 20 \mathrm{mg}$ \\
$\geq 75$ & $500 \mathrm{mg} / 20 \mathrm{mg}$ & $500 \mathrm{mg} / 20 \mathrm{mg}$
\end{tabular}

${ }^{1}$ Based on typical day-to-day fluctuations in body weight, a $\pm 3 \%$ window was permitted and used at the discretion of the Investigator when assigning the initial dose group. ${ }^{2}$ Study drug dose, given twice daily. 


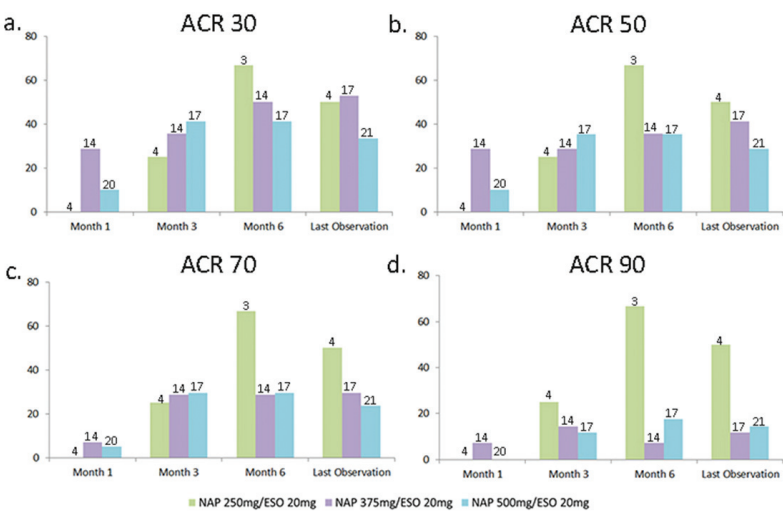

Abstract AB1086 - Figure 1

Note: the number above each bar represents the number of patients at that dose. The ACR Pediatric-30,-50, -70 , and -90 responses were defined as an improvement of at least $30 \%$ (or $50 \%, 70 \%, 90 \%$ respectively) from baseline in at least 3 of the 6 signs and symptoms variables, with no more than 1 of the remaining variables worsening by $>30 \%$. JIA signs and symptoms variables: physician's global assessment of disease activity, CHAQ disability index score, CHAQ global assessment of well-being, number of joints with active arthritis, number of joints with limited range of motion, serum CRP or ESR.

Conclusions: Improvement in JIA signs and symptoms occurred at most assessments and by month 6, the percentage of patients with an ACR Pediatric-30,$50,-70$, and -90 Response was $47.1 \%, 38.2 \%, 32.4 \%$, and $17.6 \%$. No new safety signals were identified for the well-characterised components of this fixed dosed JIA treatment, which was developed to reduce the risk of gastric ulcers.

\section{REFERENCES:}

[1] Petty RE, et al. J Rheumatol 2004:31:390-392

[2] Rigante D, et al. Clinic Rev Allerg Immunol 2015; 49:253-261

[3] Ringold S, et al. Arthritis Rheum 2013; 65 (10):2499-2512

Disclosure of Interest: D. Lovell Grant/research support from: National Institutes of Health, NIAMS. Cincinnati Childrens Hospital Medical Centre receives funds from AstraZeneca, Bristol-Myers Squibb, AbbVie, Pfizer, Roche, Novartis, UBC, Forest Research Institute, Horizon Pharma, Johnson and Johnson, Biogen, Takeda, Genentech, GlaxoSmithKline, Boehringer Ingelheim, Celgene, and Janssen for consulting, Speakers bureau: Genentech and Bristol Meyers Squibb, J. Dare Grant/research support from: AbbVie, AstraZeneca, Bristol-Myers Squibb, Horizon Pharma, Medac, Pfizer, Roche and UCB, J. Ball Shareholder of: Horizon Pharma USA, Inc., Employee of: Horizon Pharma USA, Inc., M. Francis-Sedlak Shareholder of: Horizon Pharma USA, Inc., Employee of: Horizon Pharma USA, Inc., B. LaMoreaux Shareholder of: Horizon Pharma USA, Inc., Employee of: Horizon Pharma USA, Inc., R. Holt Shareholder of: Horizon Pharma USA, Inc., Employee of: Horizon Pharma USA, Inc.

DOI: 10.1136/annrheumdis-2018-eular.1089

\section{AB1087 PROLONGED RESPONSE WITH TUMOUR NECROSIS FACTOR ALFA INHIBITION IN A 5 YEAR OLD BOY WITH SEVERE MANIFESTATIONS OF IL-36 RECEPTOR ANTAGONIST DEFICIENCY (DITRA)}

D. Clemente ${ }^{1}$, J.C. López Robledillo ${ }^{1}$, A. Torrelo ${ }^{2}$, A. Hernández ${ }^{2}$, E. Villalobos ${ }^{3}$. ${ }^{1}$ Pediatric Rheumatology Unit, ${ }^{2}$ Pediatric Dermatology, ${ }^{3}$ Pediatrics, Hospital Niño Jesús, Madrid, Spain

Background: Deficiency of the interleukin (IL) - 36 receptor antagonist (DITRA) is an autosomal recessive autoinflammatory syndrome caused by mutations in the IL36RN gene. Clinical manifestations of DITRA include recurrent episodes of generalised skin postulation, fever, systemic inflammation and leukocytosis. An uniformly effective treatment for DITRA has not yet been identified.

Objectives: We present a case of a 5 year old patient with DITRA with prolonged response with tumour necrosis factor alfa inhibition with adalimumab.

Methods: A five-year-old came to our dermatology clinic after worsening of a previous diagnosed plaque psoriasis, with an erythematous scaly dermatitis that extended throughout the trunk. Treatment with acitretin and cyclosporin were not effective and patient developed in few weeks a generalised erythroderma with pustules covering almost every part of his body, including palms and soles. He was admitted for the onset of fever and irritability due to painful rubbing of the skin. Family history of recurrent fevers or psoriasis were not revealed. Parents were not consanguineous.

Complete blood count showed leukocytosis with neutrophilia and thrombocytosis with an erythrocyte sedimentation rate (ESR) of $6 \mathrm{~mm} / \mathrm{hr}$ and a C-reactive protein (CRP) of $8,4 \mathrm{mg} / \mathrm{dl}$. Biochemistry panel revealed a mild elevation of liver enzymes without other abnormalities. Antinuclear antibody (ANA) and rheumatoid factor were negative with normal serum immunoglobulin and complement. Blood culture grew E. Coli, S. Maltophila and S. epidermidis. Skin biopsy showed acanthosis and papillomatosis with perivascular polymorphous inflammatory cells. Genetic analyses showed a homozygous mutation in the IL36RN gene (pSer113Leu). No mutations were detected in IL1RN and CARD 14 genes.

Results: Treatment was initiated with intravenous methylprednisolone $2 \mathrm{mg} / \mathrm{kg} /$ day and subcutaneous anakinra $2 \mathrm{mg} / \mathrm{kg} /$ day. Cefotaxime and co-trimoxazole were added until blood cultures were negative. Although skins lesions improved during the following days and patient was finally discharged, symptoms reappeared when decreasing the steroid dose. Three months later adalimumab and methotrexate were started, allowing the patient to end treatment with corticoids without evidence of activity of the disease.

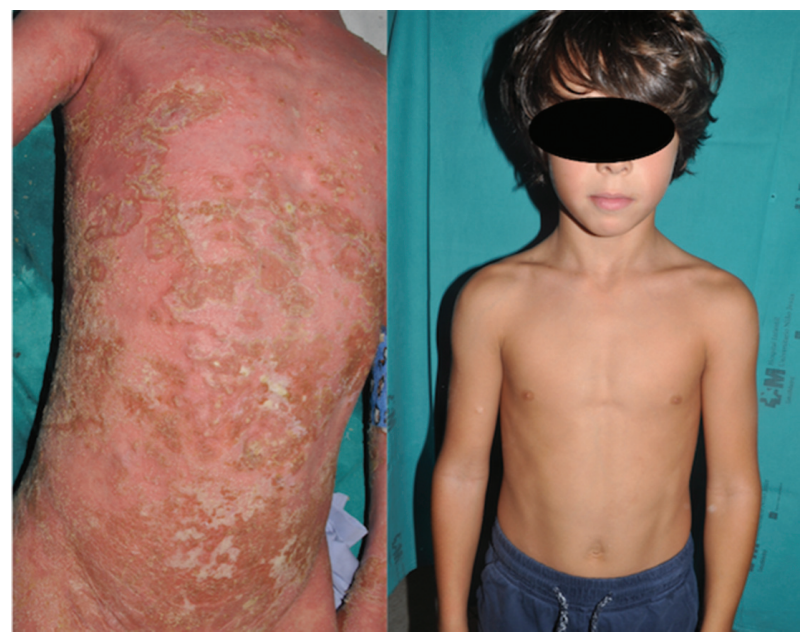

Abstract AB1087 - Figure 1

Conclusions: After incomplete response with anakinra, inhibition of tumour necrosis factor alfa resulted in a prolonged response in our patient with deficiency of the interleukin (IL)-36 receptor antagonist (DITRA).

Disclosure of Interest: None declared

DOI: 10.1136/annrheumdis-2018-eular.6532

\section{AB1088 CAPILLARY HEMOSIDERIN DEPOSITS OR EXTRAVASATIONS: A SUBTYPE OF HAEMORRHAGETHAT ACQUIRES SEPARATE ATTENTION IN QUANTITATIVE ANALYSIS OF NAILFOLD CAPILLAROSCOPY IN CHILDHOOD-ONSET SLE}

D. Schonenberg-Meinema ${ }^{1}$, M. van den Berg ${ }^{1}$, A. Nassar-Sheikh-Rashid ${ }^{1}$, M. Boumans ${ }^{2}$, M. Cutolo ${ }^{3}$, T. Kuijpers ${ }^{1}$, V. Smith ${ }^{4}$, on behalf of EULAR study group on microcirculation in rheumatic diseases. ${ }^{1}$ Pediatric Hematology, Immunology, Rheumatology and Infectious diseases, Emma Childrens Hospital, Academical Medical Center (AMC); ${ }^{2}$ Department of Clinical Immunology and Rheumatology, Academical Medical Center (AMC), Amsterdam, Netherlands; ${ }^{3}$ Research Laboratory and Academic Unit of Clinical Rheumatology, University of Genova, Genova, Italy; ${ }^{4}$ Department of Rheumatology, Ghent University Hospital, Ghent, Belgium

Background: Quality of images in nailfold capillaroscopy has improved in the last years by introduction of videocapillaroscopy. Microangiopathy, as observed in capillaroscopy of SLE-patients, ${ }^{1-3}$ can now be described by more detailed quantitative analysis. Recently, in a small cohort $(n=22)$ of childhood-onset SLE (cSLE), we described capillary bleedings by two different subtypes: large haemorrhages and small point-shaped haemorrhages with a total count of resp. 0.2/1.5 per 